\title{
Orofacial Myofunctional Therapy in Tongue Thrust Habit: A Narrative Review
}

\author{
Sejal S Shah ${ }^{1}$, Meenakshi Y Nankar ${ }^{2}$, Vikas D Bendgude ${ }^{3}$, Bhagyashree R Shetty ${ }^{4}$
}

\begin{abstract}
Aim and objective: The respective review article is to provide an overview of the various exercises in orofacial myofunctional therapy (OMT) as a treatment modality for tongue thrust habit. Tongue thrust is the persistence of an infantile swallow pattern during late childhood. This leads to breathing and speech difficulties, open bite, and protruded teeth. During formative years, most children successfully transition from an infantile to a mature swallowing pattern. However, a few develop a retained infantile swallow and tongue thrust habit which could be due to abnormal habit like thumb sucking or an underlying cause like enlarged adenoids. Adverse effects of these habits can be avoided by early detection and intervention in a growing child. Tongue thrust can be treated in different ways with early diagnosis, removal of underlying causes, correcting tongue posture, and breaking of habit with the use of orthodontic appliances. This review article is focused on the various OMT techniques employed for the correction of tongue thrust. There are several exercises in OMT which can help a child with tongue thrust. These can be performed at home under the supervision of the child's parents. Orofacial myofunctional therapy has provided a dramatic and positive influence on patients treated for tongue thrust. The joy of eating, speaking, and correct breathing can be regained along with confidence, self-esteem, and improved quality of life. Clinically, OMT plays a positive role by not only improving swallow but also the posture of tongue, improper muscle function, and reduces relapse of previous orthodontic treatments.
\end{abstract}

Keywords: Myofunctional therapy, Tongue dysfunction, Tongue habits, Tongue rest posture.

International Journal of Clinical Pediatric Dentistry (2021): 10.5005/jp-journals-10005-1926

\section{BACKGROUND}

\section{What is Tongue Thrust?}

Tongue thrust is a term that describes a swallowing pattern in which an individual pushes the tongue against or between the teeth. This habit has also been called deviate swallow, deviant deglutition, reversed swallow, perverted swallow, oral myofunctional disorder, visceral swallow, infantile swallowing pattern, and abnormal swallow. Tulley defines it as 1969-the forward movement of the tongue tip between the teeth to meet the lower lip during deglutition and in sounds of speech so that the tongue becomes interdental. ${ }^{1}$

\section{When to Correct Tongue Thrust?}

Tongue thrusting is normal in the neonate in which the tongue lies between the gum pads and the mandible is stabilized by facial muscles during a swallow. This gradually disappears with an eruption of primary dentition. Normal mature swallow shows the positioning of the tongue high on the palate behind the maxillary incisors and no lip and cheek activity during swallowing. A transitional swallowing pattern is seen in mixed dentition, when some primary teeth are lost and permanent are yet to erupt or are erupting. This type of swallow is self-correcting. In cases of open bite mostly created by habits like thumb sucking, the tongue gets thrusted ahead to achieve a lip seal. This type of tongue thrust is called a "Simple Tongue Thrust". "Complex tongue thrust" is seen in individuals with a diffuse open bite, seen most commonly in mouth breathers and in a child with a history of chronic nasorespiratory disease/allergies. "Retained Infantile Swallow" is when the infantile swallowing reflex perseveres after the eruption of permanent teeth. Intervention by a pediatric dentist is only required in cases of simple and complex tongue thrust as well as in "Retained Infantile Swallow".
1,2,4 Department of Pediatric and Preventive Dentistry, Dr DY Patil Dental College and Hospital, Pune, Maharashtra, India

${ }^{3}$ Department of Pedodontics and Preventive Dentistry, Dr DY Patil Dental College and Hospital, Pune, Maharashtra, India

Corresponding Author: Meenakshi Y Nankar, Department of Pediatric and Preventive Dentistry, Dr DY Patil Dental College and Hospital, Pune, Maharashtra, India, Phone: +91 9975607170, e-mail: meenakshi. nankar@dpu.edu.in

How to cite this article: Shah SS, Nankar MY, Bendgude VD, et al. Orofacial Myofunctional Therapy in Tongue Thrust Habit: A Narrative Review. Int J Clin Pediatr Dent 2021;14(2):298-303.

Source of support: Nil

Conflict of interest: None

This review aims to act as a reference guide for clinicians enlisting the various myofunctional training methods for tonguethrusting habit. It also describes the patient selection for orofacial myofunctional therapy (OMT) and cautions against the limitations of the same.

\section{Review Results}

Treating Tongue Thrust

Management of tongue thrust includes: ${ }^{2}$

- Habit-breaking appliances like tongue cribs act as reminders and restrict the forward movement of the tongue.

- Correction of malocclusion.

- Myofunctional therapy to correct the position of the tongue at rest and during swallow. 
All these management strategies go hand in hand. A habitbreaking appliance only attempts to block the heavy force of swallowing, which, even when added up, only amounts to about 20 minutes a day. ${ }^{3}$ The light, constant pressure of the tongue and lips have much more influence on oral equilibrium than tongue thrust ever will. Only treating the intermittent heavy force created by tongue thrusting is not treating the source of the problem and hence after removal of the habit correction appliance and the reason of the tongue thrust is not attended to, the forward tongue position and functions can return leading to relapse.

\section{Myofunctional Therapy}

Orofacial myofunctional therapy includes exercises of the cervical and facial muscles for the improvement of proprioception, tone, and mobility. ${ }^{4}$

Orofacial myofunctional therapy is neuromuscular re-education of the muscle function to improve the functions of swallow, tongue, oral breathing, and rest posture of lips, tongue, and cheeks. ${ }^{5}$

Orofacial myofunctional therapy acts as an adjunct to orthodontic treatment and helps in harmonizing the orofacial function. $^{6}$

It includes exercises that help with toning and resistance training of the oral musculature. ${ }^{7}$

Orofacial myofunctional therapy in tongue thrust habit acts as an adjunct to speech therapy and orthodontic correction of malocclusion. ${ }^{8}$ It also helped stabilize the treated malocclusion ${ }^{9}$ and improve the child's oral awareness. ${ }^{10}$

Orofacial myofunctional exercises for tongue thrust:

- Lip exercises:

- Lip closure and competency exercise

Tightly closing the lips together. The child holds a piece of cardboard/ice cream stick between the upper and lower lip for 5 seconds and repeated 5-10 times. This will improve lip competency.

- Lip puffing exercise

The child is asked to force air in-between the lips and teeth and puffing out the lips as much as possible (Fig. 1).

- Lip movements and "oo-ee" sound

The child is asked to spread the lips and purse them as in an exaggerated "oo-ee".

- Ballooning exercise

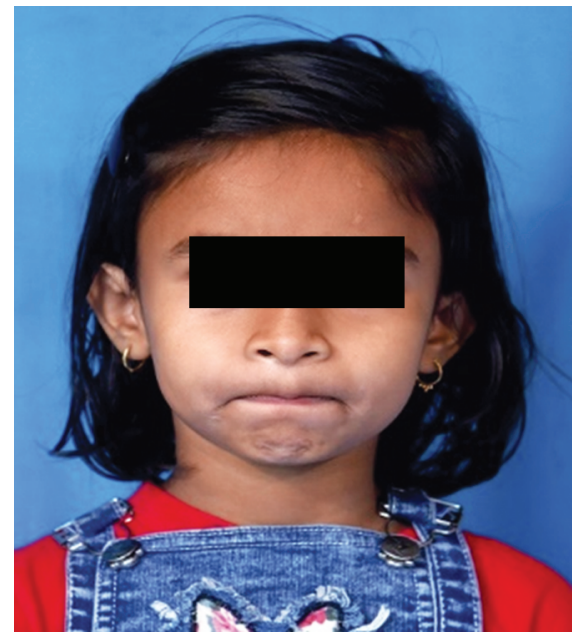

Fig. 1: Lip puffing exercise
The child is asked to blow into a balloon till its full capacity, followed by letting the air release and then repeating again (Fig. 2).

- Bubbles-blowing exercise

A child blows bubbles from a toy with help of lips.

- Button-pull exercise

A thread/string is passed through a big button of about 2-3 $\mathrm{cm}$. The patient is asked to keep the button between lips and teeth. The thread is pulled outside at the same time the button is being held tightly in place by the lips. This improves the strength of the lips.

Two buttons can be used. One of the buttons is placed between the lips of the patient, while the other is held by the patient outside.

\section{- Whistling}

The child can use a whistle as a prop or purse the lips together for whistling. Whistling activates the perioral and cheek muscles. ${ }^{11}$

\section{- Lip-stretching exercise}

The patient stretches the upper lip in a down and out motion till its maximum capacity. This is repeated 10 times. Variation of the same exercise is to hold the stretched upper lip down by pressing the lower lip against it for 30 seconds and repeated 15-20 times a day. This exercise improves the tone of the upper lip. ${ }^{12}$

- Gum drop exercise

"Gum drop exercise" in which a large size gum drop is taken, approx. 1 ounce. An 18-20 inch string, depending on the height of the patient is taken. One end of the string is attached to the gum drop, and the other end is behind the teeth and held with the lips. The patient is asked to place his/her hands behind the back and to bend forward till the face is parallel with the floor and the string is stretched. Then, the patient is asked to open his lips and stretch them as far down as possible, and the same procedure is followed 5-10 times a day.

- Card-pull exercise

In "card pull exercise" a piece of paper or cardboard is held tightly between the upper and lower lip. The child tries to pull the cardboard in an outward motion, the lips try to resist the same. It is similar to "tug of war" and "button pull exercise". 12

- Drinking out of a straw

Drinking from a straw is an exercise that shows high compliance with children which aids in oral posture correction exercising lip, cheek, and tongue muscles. However, only a small segment

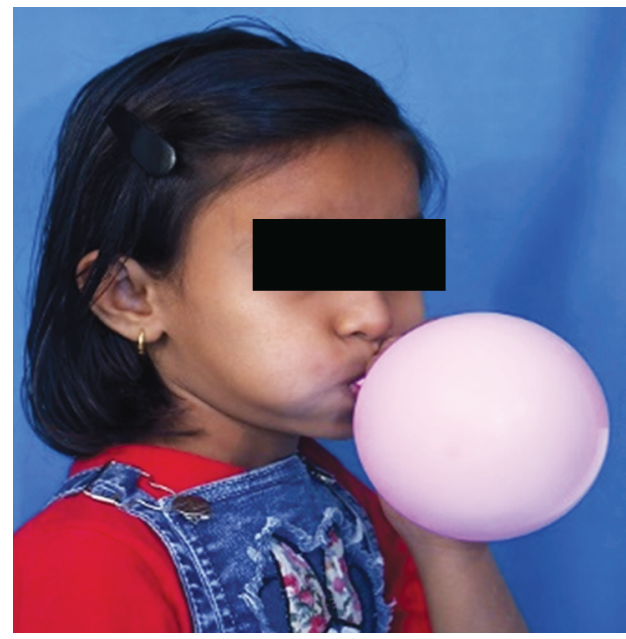

Fig. 2: Ballooning exercise 
of the straw should be allowed inside the mouth. A "Lip Blok" aids this activity where it acts as a stopper.

The impact of these exercises can be increased by using twisted straws and thick liquids like milkshakes that demand more effort to suck.

- Spoon-holding exercise with lip

The child is asked to hold the handle of a spoon between the lips, just like in a lemon-spoon race, only this time the spoon is not held by teeth but only with the help of lips. The spoon should be parallel to the floor. As the strength of the lips improves, the weight on the spoon can be increased. Repeat 10 times.

\section{- Exercise by wind instruments}

The use of wind instruments like trumpet strengthens the lip muscles and assists in confining the action of the tongue within a definite area. The supply of blood to that part of the lips coming in contact with the mouthpiece improves due to a stimulating effect. Regular and monitored usage of this instrument will result in improved tonicity of the hypotoned tissue and lengthening of short flabby lips. The position of the tongue during the usage of the wind instrument is high in the palate which is desirable, as it stimulates tissues of the lower lip while reducing tension in the upper lip.When a child plays other wind instruments like the flute, the upper, and lower lips are engaged, thereby improving the strength and tone. ${ }^{13}$

- Other lip exercises

- Suck in the cheeks and lips to make the lips look fish face. Hold this position for 5 seconds.

- Hold the lips into an "O" shape.

- Open the mouth wide to open out the lips wide apart.

- Lip KISSES.

- Make child line up stuffed animals and kiss them all goodnight.

- Tongue exercises

- Tongue spot

The child is asked to locate the spot behind the upper incisors on the palate. Hold the tongue at the spot for 10 seconds and repeat 10 times.

- Tongue click

Placing the tongue against the roof of the mouth (at the spot) snap it down, to make a clicking or popping noise.

- Swallowing thin liquid

The child is asked to take some water in the mouth, at the same time holding the tongue at the spot, not allowing the liquid to fall out of the mouth. Hold for 5 seconds and swallow. The same can be repeated by closing the teeth together followed by swallowing. In this, the tongue surface touches the roof of the mouth. ${ }^{14}$

- Swallowing thick liquid

Ask the child to take a sip of a thick liquid like a milkshake and hold the tongue at the spot, not allowing the liquid to fall out of the mouth. Hold for 5 seconds and swallow.

- 45 exercise

4S stands for spot, salivate, squeeze, and swallow. The tongue is taken to the "Spot", behind the maxillary incisors, patient is asked to hold the tongue there as he salivates, followed by squeezing the spot and then swallowing with the teeth brought together while maintaining the tongue at the position. It is advisable to follow this swallowing exercise at least 40 times a day to imbibe this new swallowing pattern. ${ }^{15}$

\section{- Touch-nose exercise}

Touch nose exercise involves protruding out the tongue and trying to touch the tip of the nose. This has to be held in this position for 10 seconds and repeated 10 times.

- Touch chin exercise

In this exercise, the child is required to protrude out the tongue to try to lick the bottom of the chin. The position should be held for 10 seconds and should be repeated 10 times.

- Tongue-sideways movement exercise

This exercise involves protruding the tongue out and moving it in extreme right and left directions. The tongue is to be held in each direction for 10 seconds. This exercise is to be repeated 10 times on each side.

- Tongue-rolling exercise

In this exercise, the child is required to roll the tongue by folding its edges toward the midline such that it resembles a taco shell. In this rolled position the child has to protrude the tongue out to the maximum extent possible while holding it for 10 seconds. This exercise has to be repeated 10 times.

- Orthodontic elastics and swallow

This exercise is done with either no. 5 or 6 elastics. The child is required to place the elastics at the tip of the tongue followed by raising the tongue to touch the spot on the palate. This exercise is to be repeated several times. While continuing to hold the elastic at the spot, the child should salivate, followed by swallowing.

- Hold and pull exercise

The hold and pull exercise is aimed at stretching the lingual frenum. ${ }^{16}$ In this exercise, the child is asked to slowly open his mouth while holding the tip of the tongue constant against the hard palate at the crest.

- Tongue-exercise using food reinforcers

Any sticky food item can be placed either on the palate, in the vestibule, corners of the mouth, or on the lips and the child licks it off with the tongue, thereby increasing the tongue strength. For these exercises, peanut butter or yogurt acts as a good reinforcer.

- K sound and swallow

This exercise involves pronunciation of the sound " $\mathrm{Kh}$ ". When

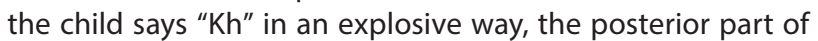
the tongue touches the soft palate and the tip of the tongue remains low. This creates awareness of the posterior tongue action in the child.K sound and swallow exercise involves first saying the "Kh" sound, followed by salivating, and then swallowing. This refines the normal way of swallowing for the child.

- Hold of tongue blades and push the tongue

This exercise strengthens the muscles of the tongue and the throat. In this exercise, the child is expected to keep two tongue blades/ice cream sticks on the incisal edge of lower anterior teeth with $2-3 \mathrm{~cm}$ of the blade extending inside the mouth. Then, the child is required to try and lift the tongue blades against the resistance of the firmly held blades (Figs 3 and 4).

- Tongue exercise using a spoon

A spoon or any hard object is to be held in front of the lips while the tongue pushes against it. This has to be done for 10 seconds with the tongue steady, straight, and not allowing it to point downward. Complete 10 repetitions of this exercise.

- Tongue retraction

In this exercise, the patient is required to touch the back of his tongue against the palate while holding it in this position for a 


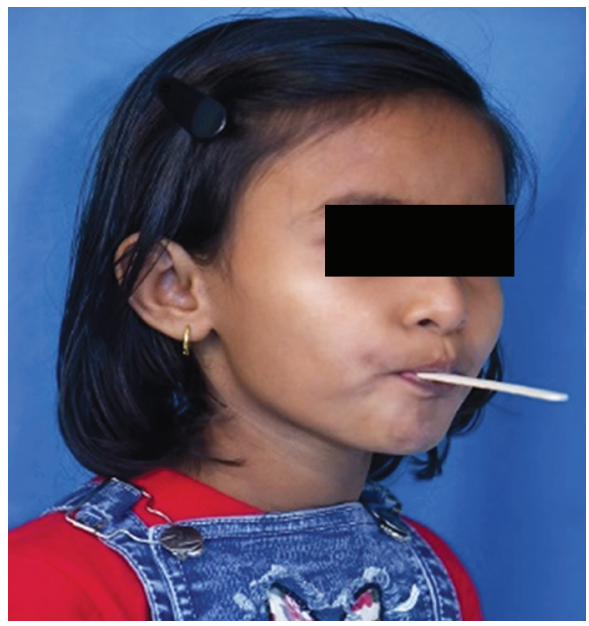

Fig. 3: Hold of tongue blades

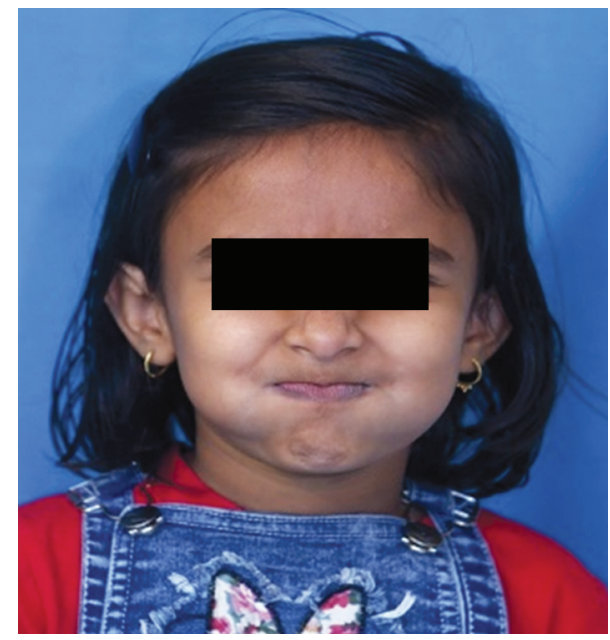

Fig. 5: Monkey face exercise

minimum of 3 seconds. This activity is to be repeated 5 times at any given instant.

- Tongue-extension exercise

In this exercise, the patient is required to protrude his/her tongue in-between the lips to a maximum extent as possible, holding it constant in this position for $3-5$ seconds. This activity is to be repeated 5 times at any given instant. ${ }^{15}$

- Tongue-pull exercise

Gently pull tongue outside and hold it with help of hands by applying light pressure. Variations: pull your tongue left, right, up, or down.

- Tongue around the world

The child is asked to move his/her tongue around the teeth in small circles (4-5 rounds) building up 15 in each direction 3 times a day to strengthen tongue muscles.

- Tongue push-ups

The child pushes his/her tongue up against a spoon/popsicle stick 10 times. He/she can try to make the tongue move straight up. While doing this exercise ask kids to look into a mirror to see whether they are doing it right or not.

- Monkey face exercise

Ask kids to put their tip of tongue over front teeth under upper lips. In the case of a full monkey face, more than a tip of the

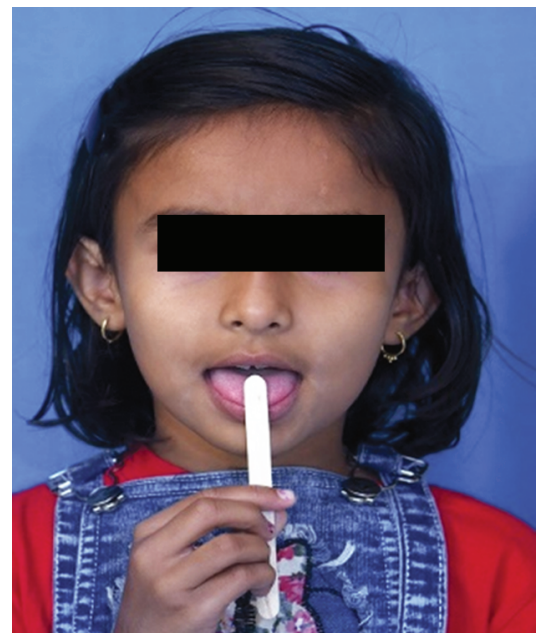

Fig. 4: Tongue push exercise

tongue covers front teeth below the upper lip. hold it for 10 seconds. This is a fun exercise and strengthens muscles (Fig. 5).

- Orthodontic rubber band exercise

First place an orthodontic rubber band on the tip of the tongue. Place the tip of the tongue against the roof right behind the upper front teeth. Occlude your teeth in a regular bite. Do not bite forward Keep the lips apart and swallow while keeping your lips apart and teeth closed Two sets of 30 swallow practices are recommended every day. ${ }^{17}$

- Lingual immaturity exercises

In this exercise, the patient has to protrude out the tongue and move it in different directions and speed. First, the patient learns to move the tongue in various directions and practices holding it steady. The next level is to move it in circular motions clockwise and anticlockwise, 10 times each. While performing circular motions the tongue tip should sweep the vermillion border of the lips. The child can perform this exercise with the help of a mirror. The difficulty of this exercise can be increased by turning the lips outward/toward the buccal surface and performing circular motions with the tongue. ${ }^{18}$

- Other tongue exercises

- Vibrating toothbrush

A vibrating toothbrush can be used for brushing teeth. The vibration stimulates tongue movement and acts as a strong sensory input.

- Teeth counting exercise

Ask children to count teeth with the use of the tongue. This helps by movements of the tongue in all the directions and challenging yet funny exercise for children (Fig. 6).

- Self-realization and swallow lessons

The child needs to ensure that the muscles used for facial expression are not engaged during swallowing for all the myofunctional training exercises listed here. The child should be made aware of the perioral, mentalis, and facial muscle contractions. $^{11}$

\section{- Air-sucking exercise}

In cases of children with open bite malocclusion, either partial, complete or thrusting of the tongue sideways, "open-lip, tongue-back, air sucking exercise" is beneficial. In this exercise, the child has to close his back teeth tightly together, open his lips wide and suck in air vigorously. At the same time, the tongue 


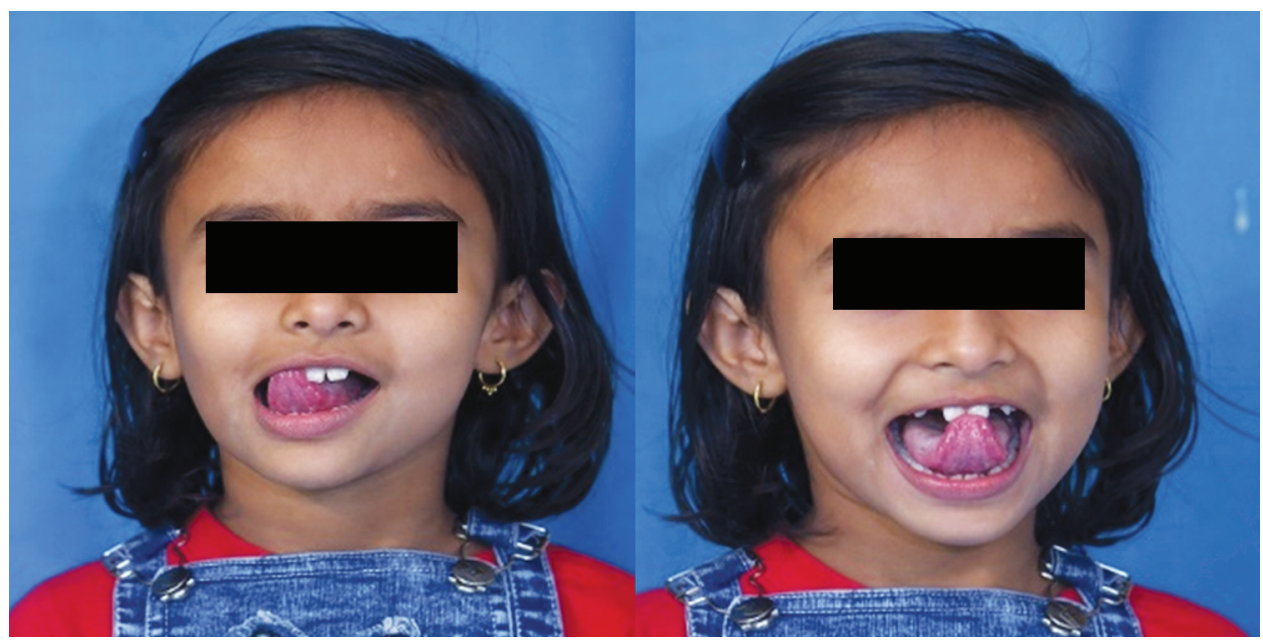

Fig. 6: Teeth-counting exercise

is to be drawn back such that the tip of the tongue touches the back of the palate ridges, followed by swallowing. A mirror should be used to ensure that the tongue is pulled back and stays in the same position without touching the teeth at any point of time or place. ${ }^{19}$

- Bite and swallow procedure

The child is asked to bite on a pliable rubber/chewie/soft tube and then by making a click sound patient is asked to bite and swallow. This activity is for improving masseter and temporalis muscle contraction.

- Cheek exercises

These exercises use a combination of water, tongue movements, and puffing to strengthen the cheek muscles.

- While holding water in the mouth the child is required to shift the water sideways.

- Alternatively, the patient can puff his/her cheeks with air. The child shifts the air from one cheek to the other while ensuring that no air escapes from the mouth or the nose. This is to be repeated a minimum of 5-10 times and should be performed 5 times a day.

- Rolling the tongue from one cheek to the other cheek.

- Fish face exercise: Puffing the cheeks with air, the child should blow a fish face repeating the activity 10 times at any given instant.

- Miscellaneous exercises

- Yawning exercise

Yawning strengthens the throat muscles as it pulls the tongue backward, upward, and also toward the back of the throat.

- Mirror exercise for the soft palate

Exercising the uvula while looking into the mirror is an effective way to improve the strength and tonicity of muscles in the soft palate and the pharynx.

- Jaw exercises:

- Handheld massagers held to the jaw for a few seconds at a time are known to stimulate the muscles of mastication. Vibration is very stimulating in short bursts and helps in relaxing the taut muscles.
- Open the jaw wide and say aaahhh!! And hold for 3-6 seconds.

- Massage the jaw gently toward and away from the lips.

- Breathing exercises

Breathing exercises include a combination of pranayam, balloon blowing, and water holding in the mouth, all while performing breathing through the nasal cavity. For pranayam, the air is inhaled and exhaled rapidly through the nose keeping the mouth shut, but relaxed. Balloon blowing exercise has a high degree of compliance from child patients. A deep breath is taken through the nose and this air is exhaled into a balloon. The balloon is expanded to its maximum capacity by exhaling more breaths into it. This can be repeated 3 times a day. ${ }^{15}$

- Subconscious therapy:

After multiple exercise sessions, reminder signs or boards can act as cues for the child to ingrain the correct swallowing pattern in the subconscious.

\section{Discussion}

\section{Patient Selection for Orofacial Myofunctional Therapy in Tongue Thrust}

Orofacial myofunctional therapy is not indicated in tongue thrusters in absence of speech/dental problems according to the authors Proffit and Mason. ${ }^{8}$ There is no common agreement in the published literature regarding the right age for starting OMT in tongue thrust. ${ }^{20}$ Some pediatric dentists have successfully used myotherapy in children $<10$ years of age. ${ }^{21}$ Whereas some prefer waiting till 10 years of age or more, as there could be spontaneous closure of the anterior open bite. ${ }^{8}$ Before starting OMT for tongue thrust patients, it is important to make sure that any anatomical variation like tongue-tie is corrected. The need for surgical reduction of excessive lymphoid tissue should also be evaluated before initiating myotherapy. ${ }^{22}$

\section{Number, Duration, and Frequency of Sessions}

A treatment program planned for any child should have a minimum of 20 sessions. The first 10 sessions are dedicated to learning the tongue posture. The next sessions help with monitoring the taught exercises. The myotherapy sessions should 
continue till the exercises are ingrained in the child's subconscious. The duration of each session should last 30 minutes. The frequency of visits can be weekly at first followed by every 15 days and later every month. ${ }^{18}$

\section{Limitations of Orofacial Myofunctional Therapy}

It is important to understand that OMT cannot replace orthodontic correction nor does it bring about changes in the morphology. It is a concept of active exercise the success of which depends critically on the motivation levels and compliance of children and their parents, equally. ${ }^{10}$

\section{Future Scope of Orofacial Myofunctional Therapy}

Successful management of tongue thrust including OMTs requires a team approach. The team includes general dentists, orthodontists, pediatric dentists, speech pathologists, and pediatricians as well. It will be crucial to bringing the attention of the clinicians to the oral musculature and its functions, which will hold the key for successful treatment of oral functions.

\section{Conclusion}

Orofacial myofunctional therapy definitely has a role in the management of tongue thrust and should be included as a part of the comprehensive treatment plan for the patient. It makes the patient aware of the faulty rest position and dynamic positions of the tongue and helps to learn the physiological myofunctional behavior. $^{10}$

\section{Clinical Significance}

Orofacial myofunctional therapy in tongue thrust helps to:

- Improve tongue elevation strength.

- Correct tongue resting posture.

- Correct tongue placement while swallowing.

- Improve tongue motility. ${ }^{10}$

- Improve maxillary constriction. ${ }^{23}$

- Prevent relapse of corrected malocclusion.

- Improve sleep-disordered breathing. ${ }^{9}$

\section{References}

1. Tulley WJ. A critical appraisal of tongue-thrusting. Am J Orthod 1969;55(6):640-650. DOI: 10.1016/0002-9416(69)90040-2.

2. American Academy of Pediatric Dentistry Guideline on Management of the developing occlusion in Pediatric Dentistry. Revised in 2009.

3. Lear CS, Flanagan JJr, Moorrees C. The frequency of deglutition in man. Arch Oral Biol 1965;10(1):83-100. DOI: 10.1016/00039969(65)90060-9.

4. Benkert KK. The effectiveness of orofacial myofunctional therapy in improving dental occlusion. Int J Orofacial Myology 1997;23:35-46.

5. Green SE. Confirmational study: a positive - based thumb and finger sucking elimination program. Int J Orofacial Myology 2010;36(1):4459. DOI: 10.52010/ijom.2010.36.1.5.
6. Homem MA, Vieira-Andrade RG, Falci SG, et al. Effectiveness of orofacial myofunctional therapy in orthodontic patients: a systematic review. Dental Press J Orthod 2014;19(4):94-99. DOI: 10.1590/21769451.19.4.094-099.oar.

7. Smithpeter J, Covell JrD. Relapse of anterior open bites treated with orthodontic appliances with and without orofacial myofunctional therapy. Am J Orthod Dentofacial Orthop 2010;137(5):605-614. DOI: 10.1016/j.ajodo.2008.07.016.

8. Proffit WR, Mason RM. Myofunctional therapy for tongue thrusting: background and recommendations. J Am Dent Assoc 1975;90(2): 403-411. DOI: 10.14219/jada.archive.1975.0075.

9. Mauclaire C, Vanpoulle F, Chaumet YSG. Physiological correction of lingual dysfunction with the "Tongue Right Positioner": beneficial effects on the upper airways. Int Orthod 2015;13(3):370-389. DOI: 10.1016/j.ortho.2015.06.007.

10. Van Dyck C, Dekeyser A, Vantricht E, et al. The effect of orofacial myofunctional treatment in children with anterior open bite and tongue dysfunction: a pilot study. Eur J Orthod 2016;38(3):227-234. DOI: 10.1093/ejo/cjv044.

11. Straub WJ. Malfunction of the tongue: part II. The abnormal swallowing habit: its causes, effects, and results in relation to orthodontic treatment and speech therapy. Am J Orthod 1961;47(8):596-617. DOI: 10.1016/0002-9416(61)90003-3.

12. Ahal R, Singh G. Interceptive orthodontic procedures. In: Singh G, ed. Textbook of Orthodontics. 2nd ed., New Delhi: Jaypee Bros; 2007. p. 564.

13. Strayer ER. Muscle instruments as an aid in the treatment of muscle defects and perversions. Angle Orthod 1938;9:18-27.

14. Maguire JA. The evaluation and treatment of pediatric oral habits. Dent Clin North Am 2000;44(3):659-669.

15. Khemka S, Thosar N, Baliga S. Oral gymnastics - way to a harmonious dentition. Int J Contemp Dent Med Rev 2015.

16. Singaraju GS, Chetan K. Tongue thrust habit - a review. Ann Essences Dent 2009;1(2):14-23. DOI: 10.5368/aedj.2009.1.2.14-23.pdf.

17. Inal $\mathrm{O}$, Arslan $\mathrm{S}$, Demir N, et al. Effect of functional chewing training on tongue thrust and drooling in children with cerebral palsy: a randomised controlled trial. J Oral Rehabil 2017;44(11):843-849. DOI: 10.1111/joor.12544.

18. Gil H, Fougeront N. Treatment of tongue dysfunction: rehabilitation for prescribers practice. J Dentofacial Anom Orthod 2018;21(4):504. DOI: 10.1051/odfen/2018137.

19. Rood MS. Neurophysiological mechanisms utilized in treatment of neuromuscular dysfunction. Am J Occ Therapy 1956;4(2):220.

20. Spreidel $T$, Isaacson $R$, Worms F. Tongue thrust therapy and anterior dental open bite. Am J Orthod 1972;62(3):287-295. DOI: 10.1016/ S0002-9416(72)90267-9.

21. Saccomanno S, Antonini G, D'Alatri L, et al. Case reports of patients treated with an orthodontic and myofunctional protocol. Eur J Paediatr Dent 2014;15(2):184-186.

22. Tarvade S, Ramkrishna S. Tongue thrusting habit: a review. Int J Contemp Dent Med Rev 2015;1(2):151214.

23. Seeman J, Kundt G, Castrillon FS. Relationship between occlusal findings and orofacial myofunctional status in primary and mixed dentition: part IV: interrelation between space conditions and orofacial dysfunctions. J Orofac Orthop 2011;72(1):21-32. DOI: 10.1007/ s00056-010-0004-1. 